\title{
Thoughts on Standard Orientation of News Gatekeeper in the Context of Modern Media
}

\author{
Hong Jiang \\ Mianyang Normal University, Mianyang, 621000, China
}

Keywords: News gatekeeper. Internet. Transmission means. News value. Context of modern media

\begin{abstract}
With the rapid development of the Internet, the channels and methods of news transmission are gradually increased, while the news gatekeepers play an important role in the news transmission, and shoulder the major responsibility. Based on the context of modern media, the author discussed the standard orientation of news gatekeeper in the modern society from five aspects of judgment on news value, selection of news angle, ability of comprehending news, application ability of news transmission means and ability of gathering news.
\end{abstract}

\section{Introduction}

The primary characteristic of modern media context is the rapid development of the Internet, which has become an important medium of news and information transmission. The recipients' roles of news and information were changed, the readers not only receive news and information, but also become transmitters of news and information, and any of the general public is likely to become transmitter of information. The rapid development of network technology and communication technology makes the new media developed rapidly, and news spread into the modern media context.

The gatekeeper in news media is the news editor, who properly cut and deeply processes the news and information they obtained, and transfer the "real" social environment to the public. Lippmann's pseudo-environment theory proposed that: the "real" social environment established in the mass transmission activities is not a mirror -style reproduction of realistic objective environment, instead, it is that the news and information is deleted, processed, reported by the news gatekeeper at a certain ideological guidance, which has a certain tendency. Therefore, the news gatekeepers' work is of profound significance. In the context of modern media, the recipients' ways and channels of getting information was increased, and the news gatekeepers need to continue to improve the quality according to actual situation to meet the diverse information needs of the recipients and promote their sustainable development. So, in the context of modern media, which aspects do the standard orientation of the news gatekeepers include?

\section{Judgment ability on news value}

In the network times, recipients' ways and channels of getting information was increased, and people are always unconscious get a lot of information in their daily lives. The network has become an essential element of people's life, and they are accustomed to using the Internet to obtain various information. The development of network media also impact on the development of traditional media to contend for the market with traditional media. However, Network media possesses characteristics of absolution responsibility and risk transmission in the transmission of news and information. Absolution responsibility means that the hierarchy of the network media gatekeeper is very simple, so that the flow of information and communication becomes freer, and the interactivity of interactive provides the recipients with the conditions of freedom of speech. Therefore, the recipients can express their views and opinions on the network, thus the recipients become the transmitters of information, as network media possesses with anonymity and invisibility, thus the information transmission has the characteristic of absolution responsibility.

As access to information on the network, the recipients are skeptical at the same time, as vast amounts of true and false information mingled with each other and the wrong and repeated messages 
on the network brought great negative impact on the recipients, especially when something was lively discussed, the mixture of true and false information can not eliminate their uncertainty, on the contrary, it will increase their insecurity, and which is not conducive to social stability and harmony.

The judgment ability on news value means that the news gatekeeper is able to discover the most newsworthy information among the numerous and complicated information at a glance, and screen the importance of the news information, that is what we often say "nose for news". In the context of modern media, the requirements on news gatekeepers' judgment ability on news value become higher. They should not only identify the most valuable information, but also screen the news authenticity and objectivity as well as screen out the most valuable information from numerous and complicated information to avoid adverse impact on society, and as far as possible to pass the "real" society to people. In addition, the Agenda Setting Theory proposed that: Although mass media can not determine the recipients' perception on a specific event, it can arrange discussion topic affect their sequence of focusing and discussing the event as well as their thinking contents.

Under the guidance of Agenda Setting Theory and pseudo-environment, as judge the value of news and information, the news gatekeepers in the context of modern media should not only judge its timeliness, authenticity, openness and novelty, but also pay attention to the following three aspects:

Firstly, educational significance of the news. In the transmission of information, the mass media not only reflects the "real" society to people, but also implies the educational significance of encouragement, teaching, and advocacy, which influenced the recipients' thinking, social ethics, values, world view, and others.

Secondly, recognizing role of the news. Let the recipients to gain the knowledge about natural sciences, meaning of life, society, etc., to improve the recipients' scientific literacy.

Thirdly, building character. Character building is not blindly catering to the recipients' tastes, but actively creating positive, optimistic, progressive and elegant tastes for them.

\section{Selection of news angle}

We all know that any event has a different point of view from a different perspective, just as the saying goes: there are a thousand readers, there must be a thousand Hamlet. The same is true for news and information, selecting the perfect angle will grace the news and receive unexpected results. The selection of news angle will also affect the value of news, so that the news gatekeeper must choose the superexcellent news angle to maximize the value of the news and information.

As choosing the news angle, news reporters are always based on their own thinking mode and life experiences, thus different reporters will choose different angles to report the same news event, and this is primary cause that the reports of different journalists are different about one same event. In the context of modern media, we live in the information environment, our surrounding changes all the time and contain much news and information, however, although we see and hear the truth, we can not extract good news angle. At that time the news gatekeeper should screen newsworthy information from distinctive angle to provide the recipients with valuable news and information.

The news gatekeeper should gather a amount of materials, grasp the social development tendency, select news reporting angle, gather valuable news materials and make comparison and analysis to discover the best reporting angle, as well as adopt corresponding expression mode, get rid of traditional conventions to provide recipients with different news and information, and realize seeing a whole through a small part and seeing the magic from ordinary by starting from common thing and details. The news gatekeeper should constantly improve their self-quality, summarize experience, and absorb others' unique perspective and reporting mode to provide readers with different news and information through perfect angle in reporting news, and strive to achieve "every one grasp the information, but no one grasp the unique angle of information reporting" to reflect the distinctive characteristics of the reports . 


\section{Ability of comprehending news}

In the context of modern media, news gatekeepers take themselves as the protectors and right defenders of the recipients' interests. They often adhere to the "reader -oriented" principle in the news reports, for example, they reflect the legibility of the manuscript as modifying the manuscript, pay more attention to the accessibility as they judging the news value, choose manuscript according to the needs of the recipients, to help readers understand the information from the layout arrangement and prompt the readers to accept the information reported by media much faster. Therefore, news gatekeepers are the connectors between the recipients and media. They permeate the social culture in their editing thoughts and editing ideas to play the social educational role of the mass media. Although the media can not directly tell the readers how to think, they can clearly tell the readers what they should think about. So, the news gatekeepers shoulder great responsibility, and they can not simply go all the way to meet the recipients, instead, they should guide the audience to think, to assume its responsibility to connect the media and the recipients.

To fully assume the responsibilities of a connector, the news gatekeepers must have certain ability to comprehend information. The news information processing procedure of the news gatekeeper is to interpret news and information, and the processing quality reflects the gatekeepers comprehending ability on news. Specifically, one of the most important skills is the critical thinking skills.

Reality is the life of news, the primary element in judging the value of news as well as the commonplace problem in academic and entity field. What is reality? What the relationship between phenomenon reality, microscopic reality and nature reality? In the context of modern media, vast amounts of information emerges in our lives, the transmissibility of the news information is strengthened, and information is easy to be deformed and distorted during transmission. In addition, the news and information itself exists inaccuracy, therefore, news gatekeeper must have good critical thinking skills to identify the authenticity of the information at the first time, preventing the wanton spread of false information. News gatekeeper must improve their quality and improve the ability to interpret news and information, judge the authenticity and nature of the information, text materials, audio, video , etc., that they saw by their critical thinking skills, inspect the reality of the events involved in the information, identify the authority of the information source, etc. extract useful information in the numerous and complicated information, remove harmful information, and then transmit the news information to the public to play the role of connector between recipients and media and protect readers' benefits and right for knowledge.

\section{Application ability of news transmission means}

In the past, the process of news transmission was: the journalist discovers the news information-the gatekeeper exams and approves the manuscripts- transmit to the recipients. In this process, the media gate keeping was easier as the media grasped almost all information sources, while the rise of network media changed the information transmission channels greatly, and the importance of traditional news gatekeeper was weakened. In the past, mass media enjoyed high authority in news information transmission while in the network environment, the authority was weakened, and the borderless and free nature of the network information transmission provided conditions for rumor breeding. Therefore, the news gatekeeper must track and monitor the comments on social networks, such as forum, blogs, etc. and timely process the false information to reduce the impact of network media. In addition, as the risks happened, the gatekeepers should integrate the information and demonstrate the valuable information in the most outstanding and concentrated place of the page, to attract readers' attention and grasp the leading positions in the network consensus.

In the context of modern media, all news media gatekeepers should guide the public opinion in the network environment to reduce the risk of public opinion. In addition, they should improve their own quality, and enhance the command ability of news transmission means.

Firstly, news media gatekeepers are not only responsible for screening news and information, but also deeply integrating the information. For instance, as an important event happened, due to the 
virtuality and concealment of the network, the audience can not distinguish the authenticity of network information, and under someone's deliberate guidance, it is possible to outbreak serious risks of network of public opinion, and cause great adverse effects even cause social panic. Therefore, it is necessary for the gatekeepers to grasp the correct positions of public opinion to prevent the spread of rumors and false information, and in addition to respect for different information and comments on the network, they should assume the responsibility to guide online public opinion, and grasp the initiative in the network of public opinion. News gatekeeper can guide public opinion hotspots by means of agenda setting, internet users symposium, expert interviews, network essay and so on, to purify network communication space.

Secondly, news gatekeeper should constantly improve their own quality, and master a variety of means of transmission. They should not only grasp the news gathering and editing in newspaper office, radio station and Television Station, but grasp the news gathering and editing in network media and mobile media. In addition, they should not only master written report skills but also master the recording, camera shooting skills as well as the audio and video editing techniques, etc.

\section{Ability of gathering news information}

In the context of modern media, media editorial staff faces thousands of news and information every day. How to find valuable information in these complicated news and information, find information related to their own interests, read and make use of the information in the limited time are important job contents of the editorial staff. If they just transmit the chaotic information to the recipients, the recipients can not perceive the intention of the transited news and information, and even at a loss as facing with the explosive information. Therefore, the gatekeepers must have the ability to gather and organize news information and recombine the valuable news and information to highlight the importance level of the news and information and impact of the readers' reading priorities.

News gatekeeper should learn to optimize the structure of the news, discover the intrinsic link between the different news and information, and arrange the structures of the news through certain transition and correlation to enhance the transmission effect of the news and enhance the expressive force of the news information. Certainly, the news must possess inherent rules and creativity, as invariable news will impact the transmission effect of the news. News gatekeepers have to understand the recipients' reading demands and study their reading psychology, and respect for the recipients through humane structural arrangements to enhance the transmissibility of the news information in that not only reflect the real news and information but also fully play the initiative of news and information and maintain the integrity of the news results, helping readers to understand the news, receiving news and information transited by media, receiving the news facts and think something under the guidance of the media.

\section{Conclusion}

All in all, the role of the news gatekeeper in the context of modern media has undergone great changes in that their responsibilities become heavier. A qualified gatekeeper must possess five basic skills. They should constantly learn new knowledge, accumulate experience, grasp a variety of skills, and organically integrate the transmission techniques of the traditional media and modern media to create a new news transmission trend in the network environment and transit social culture. In addition, to help recipients to establish cognition about society and world through daily trivial news information to exert the social educational function of mass media and influence people's life pattern, value, and so on.

\section{References}


[1] Liao Qiuhong. Interpretation on the Role of News Editors in the Modern Transmission Context, Modern Communication, 2010 (7).

[2] Zhang Jinglong, Pan Haitao. Standard Orientation of the News Gatekeeper in the Modern Transmission Context, Press Circles 2012 (17).

[3] Sheng Fang. Untrue News and Media Culture Variation in the New Media Context, Editors Bimonthly 2012 (3).

[4] Shi Changshun. The Reconstruction of News Media Role under Media Integration Context, Academic Journal of Zhongzhou 2010 (6).

[5] Huang Weicheng, Chen Xinyong. The Role of Network Editor in Risk Transmission, Today 'S Mass Media 2009 (5). 